\title{
eLight: enlightening and exploring light
}

\author{
Aydogan Ozcan ${ }^{1 *}$ and Cheng-Wei Qiu ${ }^{2 *}$
}

Optics and photonics-enabling the human kind to answer basic questions such as "how can people see things"- has now evolved to be a main-stream discipline rather than a sub-topic of physics.

In the past decades, more than 40 Nobel Prizes are awarded to optics-related researches. With UNESCO designating 2015 and May $16^{\text {th }}$ as the International Year of Light and International Day of Light, respectively, we have witnessed how optical sciences and engineering of light prospered into a game changer in the wellbeing of human society, reshaping and fusing various disciplines including physics, biology, chemistry, biomedical sciences and engineering. Optics and photonics research has fundamentally reformed our understanding of the nature, and all these transformative advances have motivated us to start a new journal to timely showcase some of the emerging groundbreaking research driven by optics and photonics.

Here we announce the launch of eLight, aiming to bring the finest and most exciting research results in optics and photonics fields, facilitating the wide dissemination of some of the most exciting scholarship on light. As a fresh and ambitious journal, we have formed:

a. An Editorial Board of world-renowned scientists and engineers, who are dedicated to select and manage the peer-review of the eLight submissions.

b. A transparent, fair and rapid peer-review process, which will publicly share the referee comments and authors' responses, under the consensus of the authors and reviewers. c. A fast publication pipeline along with multiple public dissemination channels, with an expected turnaround period of 60 days. Each accepted paper will be accompanied by a Commentary and the results will be widely shared with the public and research community through different press release channels, in a timely manner.

eLight is co-published by Changchun Institute of Optics, Fine Mechanics and Physics, Chinese Academy of Sciences and Springer Nature, and is managed by the same team which created the journal Light: Science $\mathcal{E}$ Applications-a leading optics journal. And eLight is selected as the China's high-profile new journal, quoting from https://www.nature.com/articles/d41586-01903770-3.

We look forward to having your contributions, and please join us to move eLight forward towards enlightening and exploring light.

\section{Authors' contributions}

$\mathrm{AO}$ and $\mathrm{CWQ}$ jointly wrote this editorial piece. All authors read and approved the final manuscript.

\section{Competing interests}

The authors declare that they have no competing interests.

Published online: 08 June 2021

\footnotetext{
*Correspondence: ozcan@ucla.edu; chengwei.qiu@nus.edu.sg

1 Electrical and Computer Engineering Department, University of California, Los Angeles, CA 90095, USA

2 Department of Electrical and Computer Engineering, National University

of Singapore, 4 Engineering Drive 3, Singapore 117583, Singapore
} 